\title{
GRAFITIS COMO ACTO DE ENUNCIACIÓN. TRAZANDO TRAYECTORIAS DE SENTIDO EN EL CAMPO DE LA INVESTIGACIÓN EN COMUNICACIÓN SOCIAL
}

\author{
GRAFFITI AS AN ACT OF ENUNCIATION. TRACING TRAJECTORIES OF MEANING IN THE FIELD OF
}

SOCIAL COMMUNICATION RESEARCH

\author{
Lucrecia A. Sotelo* \\ lucreciasotelo@gmail.com
}

RESUMEN: Este artículo tiene la finalidad de poner en cuestión aspectos centrales que hacen a la investigación de la comunicación social y, con ello, de la cultura. Por tanto se parte de la investigación realizada dentro de la materia "Teorías de la Comunicación 1" donde, los estudiantes junto a la docente, realizaron un estudio exploratorio en torno a los "grafitis" y su dimensión enunciativa. La actividad se llevó a cabo en la ciudad de Caleta Olivia, provincia de Santa Cruz, Argentina durante los meses de abril a julio del año 2016. Desde la construcción de la Unidad de Análisis hasta la aplicación del proyecto se trabajó desde la "teoría fundamentada" y "trayectorias de sentido". En este artículo, por tanto, se detendrá la mirada en el proceso que condujo a la construcción de la unidad de análisis. Excede, a los límites del mismo el desarrollo de la investigación. Sin lugar a dudas ello conducirá a un nuevo artículo

PALABRAS CLAVE: Comunicación Social, Cultura, Trayectorias de Sentido, Cartografías.

ABSTRACT: This article has the purpose of questioning central aspects that make the investigation of social communication and, with it, of the culture. Therefore it is part of the research carried out within the subject "Theories of Communication 1", where students, together with the teacher, carried out an exploratory study on "graffiti" and its enunciative dimension. The activity was carried out in the city of Caleta Olivia, province of Santa Cruz, Argentina during the months of April to July of the year 2016. From the construction of the Analysis Unit until the application of the project was worked from the " "And" trajectories of meaning ". In this article, therefore, it will stop the look at the process that led to the construction of the unit of analysis. It exceeds, to the limits of the same the development of the investigation. Undoubtedly this will lead to a new article.

KEYWORDS: Social Communication, Culture, Sense Trajectories, Cartographies.

\section{Introducción}

"El hombre ordinario ya no ríe", dice Michel De Certeau leyendo a Freud. No ríe - y sostiene elevando el tono de sus palabras - porque se haya subsumido con la muchedumbre cuyo destino común es ser engañada, frustrada, obligada a laborar, sometida por tanto a la ley de la trapacería y el trabajo de la muerte.

\footnotetext{
* Licenciada en Comunicación Social por la Universidad Nacional de Córdoba y Doctora en Comunicación Social por la Universidad Nacional de la Plata. Es docente en la Universidad Nacional de la Patagonia Austral.
} 
El hombre ordinario transcurre por las calles de esta modernidad tardía; interpelado como común, se resiste y traza su singularidad; busca los intersticios que la sociedad mediatizada va dejando libre.

El hombre ordinario emerge y toma el espacio; conquista el blanco insondable de las paredes; y allí, justo allí donde nadie imagina, imagina; allí donde lo blanco es limpio él crea y recrea con colores. Son los grafitis, devenidos tras las manos de jóvenes, los que enuncian una advertencia: yo estoy aquí. Expresión que se erige desafiando lo estatuido desde el lugar del narrador que nombra la realidad dando existencia. Afirmación que grita desde el centro de tu ciudad para que lo puedas, así, ver.

Los grafitis se erigen como discursos narrados en paredes, desde hace miles de años. Los hubo en impresiones negativas en cuevas como en murallas que dividen mundos, ideologías y autoritarismos. El grafiti es una de las tantas formas que asume el enunciado para nombrar la cultura. Y, como plantea De Certeau: “... el acceso a la cultura comienza cuando el hombre ordinario se convierte en el narrador, cuando define el lugar (común) del discurso y el espacio (anónimo) de su desarrollo..." (DE CERTEAU, 2010, p. 9) Es decir, el acceso a la cultura se da cuando la práctica se torna en productora de sentido, es decir, en texto. Los "grafitis", entonces, son un texto expresado en un discurso visual. Por tanto, su lectura permite dar cuenta de una determinada cultura; y es determinada, es decir, es esta y no otra, porque ellos cuentan una historia situada.

En virtud de ello, en este artículo se pretende compartir la investigación que se llevó a cabo con estudiantes de segundo año de la carrera de Comunicación Audiovisual de la Universidad Nacional de la Patagonia Austral el año 2016. Dicha investigación fue un estudio exploratorio, por tanto "no consistió en sustituir con una representación o encubrirla con palabras irrisorias, sino en mostrar cómo se introduce en nuestras técnicas (...) y puede reorganizar el sitio donde el discurso se produce" (DE CERTEAU, 2010, p. 10)

Cabe destacar que este estudio se constituye, por un lado, como una primera aproximación al campo de estudio en Caleta Olivia, provincia de Santa Cruz - Argentina - y, por el otro, se presenta como la primera experiencia de investigación en comunicación que los estudiantes realizan dentro de su formación de grado. Aquí se buscó interrogar el campo y asir a la comunicación como práctica específicamente significante. 
En este sentido, Pierre Bourdieu, en ¿Qué significa hablar? plantea que "...no existe nada que no se pueda nombrar y se puede nombrar la nada". (2008, p. 17) De manera que, lo que aquí se busca es ir tras esas palabras que tienen el poder insondable de nombrar aquello que es invisibilizado. Palabras en colores. Palabras en paredes, que no son otra que las imprentas de los pueblos.

Atendiendo a lo expuesto, en este artículo se presentará la unidad de análisis que condujo al planteo de la investigación. En su desarrollo se irán articulando aspectos fenomémicos que permitieron interrogar el objeto de estudio. Por tanto, en este texto se presta detenida atención a los aspectos teóricos pero fundamentalmente metodológicos que atraviesan la constitución de la investigación en el campo de la comunicación social.

Se habla de grafitis, si; se habla de grafitis como objeto de estudio; por tanto, lo que aquí se pretende es poner en cuestión a la comunicación como ciencia.

\section{Cartografías de la investigación. Sobre lo pretendido y lo aprendido}

Diseñado como estrategia pedagógica pero anclado en el necesario desafío de dar cuenta al movimiento, se planteó dentro del espacio curricular "Teorías de la Comunicación 1" el desarrollo de una investigación empírica de carácter exploratorio. Como tal, se encuentra organizada desde los siguientes interrogantes:

- ¿Qué se realizó? ¿Cuáles son las acciones que se llevaron a cabo?

- ¿Cuáles son las bases que fundamentan las acciones emprendidas?

- ¿Qué metas se persiguieron?

- ¿Quiénes participaron?

- ¿Qué críticas se pueden realizar al proceso emprendido?

- ¿Qué quedó afuera de la investigación? o ¿Qué no se pudo lograr?

- ¿Qué nuevos desafíos abre esta investigación?

El punto de partida de esta investigación estuvo dado en la necesidad de tornar asequible en la vida cotidiana, los elementos que definen y constituyen a la comunicación como ciencia. De manera que, el diseño y ejecución de este proyecto nació como estrategia pedagógica para situarse como un espacio central del proceso de aprendizaje dialógico entre los estudiantes y los diferentes enfoques teóricos que tienen a la comunicación como objeto de estudio. 
En virtud de dar cuenta del camino diseñado, se estructuró el proyecto de investigación atendiendo a los siguientes ítems:

a- Definición del área problemática: exploración teórica y definición de la unidad de análisis.

b- Definición metodológica del estudio exploratorio. Elaboración de las dimensiones de análisis, diseño de las herramientas. Especificación de las entradas al campo. Planteo de las entrevistas en profundidad. Procesamiento de la información. Cartografía y trazado de trayectorias de los grafitis.

c- Elaboración del análisis. Identificación de espacios de encuentro; determinación de zonas de análisis.

El planteo de cada uno de estos ítems partió de una estrategia fundamental: la problematización de los nudos teóricos de las diversas teorías de la comunicación a partir de un observable que constituye la vida cotidiana de los estudiantes. Es decir, se pretendía poner en cuestión "lo cotidiano" en pos de tornar evidente aquello procesos que significan lo social. El camino tque se siguió estuvo guiado por la enfoque de la "teoría fundamentada" y la estrategia de análisis de la perspectiva teórica de las Trayectorias de Sentido. (SOTELO, en prensa)

\section{Definición del área problemática}

El puntapié inicial que articuló el eje problematizador de la investigación fue el interrogante: ¿qué es la comunicación? Ante esta situación, la respuesta comenzó a darse desde la teoría y se constituyó en marcado disparador. Asumiendo ese desafío se definió a la comunicación como:

Un conjunto de intercambios que construyen la red discursiva de la sociedad. Esta red es tejida por las prácticas productoras de sentido de los agentes sociales (individuos, instituciones, objetos) que se expresan en los discursos.

Esta definición implica un marcado posicionamiento teórico que pone en discusión varios conceptos. Siendo así, la comunicación es considerada desde la ponderación de la relación que se establece entre los agentes, las instituciones y los objetos. Esa relación se da de manera compleja ya que parte desde el punto vista/ visión de mundo de todos aquellos que participan en ella. Es relación, por tanto, es intercambio. Este intercambio se da desde el reconocimiento de las disputas de los agentes por nombrar el orden social. Visiones de 
mundo puestas en juego. Visiones de mundo devenidas en las trayectorias de los agentes significadas por la puesta en juego de las prácticas de los agentes en el espacio social. Visiones de mundo que disputan por el poder nombrar; disputas plausibles de ser advertidas en los discursos.

Considerar esta perspectiva de la "comunicación" Ileva a tener en cuenta elementos dispares y similares. Considerar a la "comunicación" como un conjunto de intercambios que constituyen la red discursiva de la sociedad implica trascender aquella visión teórica centrada en la transmisión unidireccional de mensajes. Aquel proceso visible en los planteos del "Modelo Informacional" el cual está centrado en el rol del emisor como difusor hacia un receptor pasivo.

En virtud de ello, resulta pertinente destacar las palabras de Eliseo Verón:

... un discurso, producido por un emisor determinado en una situación determinada, no produce jamás un efecto. Un discurso genera, al ser producido en un contexto social dado, lo que podemos llamar un campo de efectos posibles. Del análisis de las propiedades de un discurso no podemos nunca deducir cuál es el efecto que era en definitiva actualizado en recepción. Lo que ocurrirá probablemente, es que entre los posibles efectos que forman parte de ese campo, un efecto se producirá en unos receptores y otros en otros. De lo que aquí se trata es de una propiedad fundamental del funcionamiento discursivo, que podemos formular como el principio de la indeterminación relativa del sentido: el sentido no opera según una causalidad lineal. (VERÓN, 1993, p. 78)

El sentido se erige indeterminado y se da en el establecimiento de un campo de efectos posibles. Ello implica que el "proceso de comunicación" no se circunscribe a lo que se expresa en la emisión. El proceso de codificación como el de decodificación se da dentro una gramática de sentido en la que confluyen las trayectorias de todos y cada quienes intervienen. Estas trayectorias refieren al movimiento que el agente lleva a cabo en el espacio social. Ese movimiento se da en el establecimiento de relaciones entre los agentes, las instituciones y los objetos. El establecimiento de estas relaciones torna evidente o visible las disposiciones de todos aquellos que actúan. Es decir, aquellas decisiones que propenden a la acción. Cada trayectoria puede ser similar a otra pero nunca igual ya que ellas son el trazado de las prácticas significantes de cada agente. Si bien ellas pueden presentar propiedades similares que permitan agruparlas en clases, nunca son iguales. Es debido a ello que se considera a la "comunicación" como esa red de intercambios de sentido que pugnan por nombrar el orden social. Disputan poder porque torna evidente las diversas $y$ 
divergentes visiones de mundo.

Frente a esta forma de conceptualizar la comunicación cabe la pregunta: ¿cómo asir dichos elementos en la vida cotidiana? Es decir, ¿qué ejemplo se puede dar para profundizar el análisis?

Sin lugar a dudas, la respuesta viene de la mano de la definición del tema y, con ello, del objeto de estudio.

\section{Definición del tema}

Pensada como disparador para el análisis, el concepto de comunicación provisto abre múltiples y variadas aristas; invita a la ahondar en la reflexión propendiendo a la comprensión compleja de los elementos constitutivos. En busca de andar ese camino, y en aras de dar cuenta el proceso de investigación indicado, el primer paso que se dio fue el que condujo a la delimitación de lo que se pretendía conocer; es decir, la definición del área problemática. No fue un camino sencillo, pero se presentó desafiante. El objetivo consistía en llevar a cabo una transposición: de lo conceptual a lo fenomenológico.

Luego de intercambios, fluidas discusiones, se atendió al desafío. Siendo así, se definió como área problemática: "Los grafitis como práctica cultural alternativa".

La elección de este tema nace de la incomodidad y de la ignorancia. ¿Qué son los grafitis? ¿Cómo se escogen los lugares para pintar? ¿Quiénes lo hacen? ¿Por qué los grafitis pueden ser abordados desde el concepto de comunicación propuesto?

Múltiples y variados interrogantes contribuyeron a delimitar este complejo escenario de trabajo. Por tanto se definió como ejes problematizadores de la acción, a dichas preguntas las cuales guiaron el estudio exploratorio constituyéndose en "metas" a seguir.

El tema escogido se define como un problema complejo. Es decir, se constituye como tal en cuanto que articula un conjunto de conceptos provenientes de diversas teorías. Por tanto, complejo no por difícil; complejo porque pone a dialogar trayectorias teóricas de matriz divergente, tan divergente como la realidad a la que pretende analizar. En este sentido, el área de estudio definida se erige como la trama de sentido que interpela a la intervención urbana como una dimensión de lo alternativo y de la cultura. El abordaje de los 
grafitis, como categoría de análisis, implica el reconocimiento de otro conjunto de conceptos tales como: juventud, identidad, estética, acto de enunciación - entre los principales-. El camino trazado, por tanto, implica dar cuenta de estos conceptos a la luz del trabajo de campo. Por tanto, la definición de este tema permitió, por un lado, poner en cuestión - y con ello problematizar - los elementos que definen a la comunicación. $\mathrm{Y}$, por el otro, hacer dialogar a este concepto con otros que se sitúan en las disciplinas de las humanidades.

Una vez advertido el tema e identificado los nudos conceptuales que lo contienen, alumnos y docente - constituidos en grupo de investigación - definieron los pasos a seguir. Por tanto, se dio paso a la elaboración del proyecto de investigación. Siendo así, se definió que tipo de estudio se iba a realizar y qué momentos compondría y qué plazos serían necesarios cumplir para poder llevarlo a cabo.

\section{Del orden del hacer. De lo teórico y lo metodológico.}

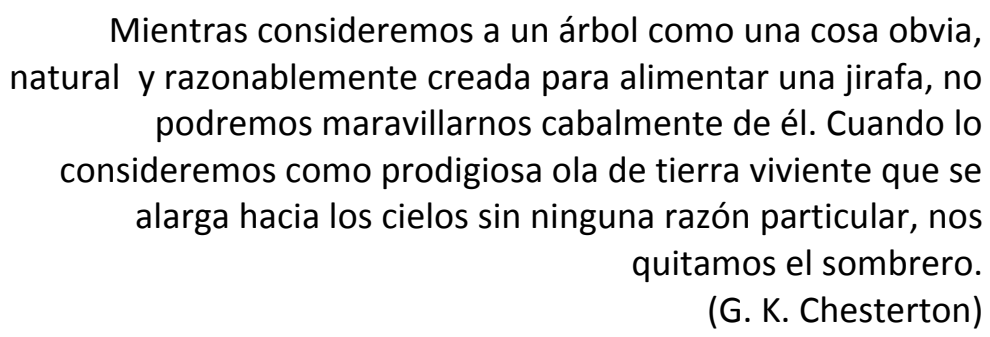

Dado que esta investigación se erige del diálogo pedagógico entre la teoría y la práctica, la estrategia de trabajo escogida conjugó: la "teoría fundamentada" y las "trayectorias de sentido". Este enfoque parte del reconocimiento de la dimensión significativa de la práctica de los agentes y sugiere un conjunto de herramientas e instrumentos que permiten dar cuenta de las dos dimensiones de la acción: la materialidad y la significatividad.

Se parte de la combinación de la "teoría fundamentada" y las "trayectorias de sentido" desde el reconocimiento de lo que las une como, fundamentalmente, desde lo que las diferencia. La elección teórica como la metodológica implica un fuerte posicionamiento en la construcción de conocimiento. Es el lugar desde el que se parte, por tanto, debe tener cierta claridad para poder tener la certeza suficiente.

En aras de explicitar el camino seleccionado, resulta de vital importancia dar algunas notas que introduzcan sobre ambas enfoques teóricos metodológicos; para así, 
siguiendo la cadencia se podrá seguir las notas del camino propuesto.

En este sentido, la "teoría fundamentada en datos" propone una metodología basada en dos estrategias: el método de la comparación constante y el muestreo teórico. $\mathrm{A}$ través del primero, se recoge, codifica y analiza datos en forma simultánea para generar teoría. Es decir, estas tareas no se realizan en forma sucesiva sino simultánea, y no están dirigidas a generar teoría, sino solo a demostrar que son posibles. Debido a ello, se llevó a cabo dos procedimientos:

- Ajuste: las categorías deben surgir de los datos y ser fácilmente aplicables a ellos; y

- Funcionamiento: deben ser significativamente apropiadas y capaces de explicar la conducta en estudio.

Tal como señala Soneira (2006) el muestreo teórico es distinto del muestreo estadístico:

... el muestreo teórico se realiza para descubrir categorías y sus propiedades, y para sugerir las interpelaciones dentro de una teoría. El muestreo estadístico se realiza para obtener evidencia precisa sobre distribuciones de una población entre categorías, que pueden ser utilizadas en descripciones o verificaciones. (GLASER Y STRAUSS, 1967, p. 62)

Mediante el muestro teórico el investigador selecciona casos a estudiar según su potencial para ayudar a refinar o expandir los conceptos o teorías ya desarrolladas. La "saturación teórica" significa agregar nuevos casos hasta que no represente información adicional por medio de la cual el investigador pueda desarrollar nuevas propiedades de las categorías.

En cuanto al enfoque: "trayectorias de sentido" se plantea que, una manera de ingresar al estudio de la realidad es ir tras las huellas de sentido; esas marcas discursivas construidas por las prácticas de los agentes; prácticas dadas en su devenir por el espacio social. De manera, que las "trayectorias" plantea que, para conocer dichas prácticas productoras de sentido, se debe diseñar un trabajo de campo que parta de la aprehensión del objeto de estudio inserto en la realidad para luego tomar distancia y así favorecer la objetualización conducente al análisis praxeológico. Por tanto, este planteo teórico metodológico propone definir una estrategia que vaya desde la dimensión fenoménica hacia una metareflexiva.

Dado que se construye desde el reconocimiento de la complejidad como eje 
estructurador del conocimiento, el enfoque de las "trayectorias de sentido" plantea que el proceso de construcción del dato no se reduce a la mirada dictamina de lo cualitativo o cualitativo. En este sentido, la forma desde la cual se considerada lo complejo discute y se aleja de cierto monismo metodológico que desconoce la estructura objetiva donde se plantea la práctica del sujeto; estructura erigida desde el reconocimiento, tal como se expresó, de las relaciones planteadas en el devenir del espacio social.

El planteo metodológico, por tanto, no puede construirse desde la linealidad, aquella que parte de la elaboración ajena al campo; aquella que fue tan común y dominó la escena de las ciencias sociales durante tanto tiempo y fundó - entre otros - los estudios comunicacionales -. La dinámica que asumen las prácticas de los agentes no responden a un orden causal ni lógico; todo lo contrario. Esa "lógica", tan mentada por un determinado paradigma de investigación, solo puede darse si se niega la complejidad desde la cual devienen las acciones. Pero y por sobre todo, cabe destacar que, ese ocultamiento o reconocimiento no se da en sí mismo, es decir, no existe en la realidad sino - como señala el Dr. Jorge González - como producto de la construcción del investigador.

En el trabajo de campo no se ven espacios sociales, ni campos educativos, ni capitales; esos espacios, campos y capitales son tales porque el investigador indica, recorta y ordena las relaciones que considera que son pertinentes para explicar el interrogante que se propone abordar dentro del proceso de investigación. Ello no implica que el investigador "manipule" sino que establece relaciones, organiza y analiza procesos.

Esta afirmación propone un reposicionamiento del investigador dentro del proceso de trabajo de campo. Su "laboratorio" no está afuera ni adentro de él. Su laboratorio es aquel "espacio" donde no se distingue, la construcción de modelos, de la observación participante abierta. Como plantea Pierre Bourdieu:

... [para] comprender sus prácticas [las del agente], es necesario reconstruir el capital de esquemas informacionales que les permite producir pensamientos y prácticas sensatas y regladas sin intensión de sentido y sin obediencia consciente... (BOURDIEU, 2007, p. 83).

De manera que no es posible abordar metodológicamente esta investigación siguiendo un modelo pre - diseñado, pero tampoco es posible avanzar sobre el camino incierto de la etnografía abierta.

Siendo así, este trabajo plantea que reconocer la complejidad, implica - a riesgo de 
parecer tautológico - reconocer al problema complejo; es decir, aquel que se haya comprendido en la interdisciplinariedad; ese que no puede ser comprendido desde una sola variable sino el que parte del establecimiento y reconocimiento de las relaciones que lo componen y definen.

El abordaje metodológico de un problema complejo requiere, no solo asir a la interdisciplinariedad como marco epistemológico sino comprender el camino de la ciencia interpretativa. Este posicionamiento sitúa al sujeto de la investigación y al investigador dentro de un espacio social que es construido por el investigador en términos del sujeto investigado. Al respecto Clifford Geertz sostiene:

El hombre es un animal inserto en tramas de significación que él mismo a tejido, considero que la cultura es esa urdimbre y que el análisis de la cultura ha de ser, por tanto, no una ciencia experimental en busca de leyes, sino una ciencia interpretativa en busca de significaciones ... (GEERTZ, 2006, p. 20)

Desde este punto de vista, las relaciones que se establecen (como indica Geertz: seleccionar informantes claves, transcribir textos, establecer genealogías, trazar mapas del área, llevar un diario, etc.) para avanzar en el camino metodológico propuesto, se tornan significativas si el camino del análisis se da dentro de un proceso analítico de descripción densa. En este sentido Clifford Geertz plantea que el análisis debe consistir en desentrañar las estructuras de la significación y, junto con ello, determinar su campo social y su alcance.

De manera que la interpretación pone en juego un complejo proceso de descripción que no se reduce a "decir" lo que se ve a simple vista. Va más allá de eso dado que, necesariamente, parte del punto de vista del sujeto de la investigación en el encuentro con las categorías de análisis que plantea el investigador. En relación a ello propone Clifford Geertz:

... en el estudio de la cultura, el análisis penetra en el cuerpo mismo del objeto (...) la línea que separa la cultura (...) como hecho natural y la cultura (...) como entidad teórica tiende a borrarse; y tanto más si la última es presentada en la forma de una descripción, y desde el punto del vista del actor, de las concepciones (...) de todas las cosas, desde la violencia, el honor, la dignidad, y hasta la justicia..." (GEERTZ, 2006, p. 28).

Siendo así, en el proceso de interpretación, se establece una relación dialéctica entre los conceptos teóricos indicados y el punto de vista del agente investigado. Es el encuentro de los puntos de vista - investigador/ investigado - el que plantea una "relación" 
entre sujetos; por lo tanto, una relación intersubjetiva que solo es dable en la praxis. Entonces, una relación que se da en dos dimensiones: una de significatividad y otra de materialidad.

Este planteo metodológico, por tanto, busca inscribir los discursos sociales dentro de las relaciones que los define y les da forma. Para ello plantea un conjunto de herramientas e instrumentos que hacen posible asir la complejidad que se persigue. El abordaje del objeto de estudio, por tanto, implica un camino por etapas que propicien una mirada compleja, desde cada punto, desde cada ángulo. Compleja, lo que no implica difícil; compleja porque no es lineal (compleja como esta estepa que nombra, allí donde se cree que no hay nada). Entonces, para comenzar a andar este camino, se diseñan diversos apartados en los cuales se abordan: los supuestos metodológicos, se desarrolla las herramientas y los instrumentos.

Antes de abordar los puntos planteados, se reflexiona sobre la situación metodológica que comprende y debate esta investigación.

Dado que esta investigación emprende el camino esbozado, resulta pertinente analizar los diversos abordajes metodológicos que ello reviste. Es decir, las bases epistemológicas que comprenden y dan forma a los modos en los que el investigador emprende el camino del conocer.

En este sentido se destaca que los dos enfoques - el de la teoría fundamentada y el de la trayectoria de sentido -,tal como se indicó, se complementan. Tienen un punto de encuentro en el proceso de construcción teórica y en la consideración del trabajo de campo. En virtud de ello, a continuación se presenta el diseño metodológico y su aplicación, situación que conduce a la elaboración teórica.

\section{Diseño metodológico}

Luego de definir y especificar el área problemática se tomó una decisión de equipo de trabajo: "Somos un grupo y trabajamos respondiendo al nosotros".

Esta afirmación enviste a la investigación en un proceso de nosotrificación. Si bien cada miembro tendría diferentes responsabilidades ello no los coloca en situación de superioridad con respecto al resto sino que, reconociendo las diferentes capacidades de cada uno se emprende el camino definido. La nosotrificación implica una visión de mundo: 
no se miran los observables con los ojos de un solo investigador; es la mirada del conjunto, desde las trayectorias de cada quien, que se aprehende la unidad de análisis construida.

Asumir la nosotrificación implica una necesaria transposición metodológica. Es decir, no se reduce a una declaración de principios de manera que hay que planificar el trabajo atendiendo a la construcción del nosotros. Este dato no es menor, dado que se tiene que tener presente que este trabajo de investigación se encuentra dentro del dictado del espacio curricular "teorías de la comunicación 1". De manera que, resultaba necesario distinguir los tiempos, las dinámicas, los espacios para poder avanzar con claridad.

Siendo así, el primer paso a dar fue la definición de una estrategia; es decir un posicionamiento de cara la investigación. En virtud de ello, la estrategia definida fue:

Reunión plenaria cada 15 día. Allí se discutían lo realizado, se analizan los plazos y se problematizan las dificultades.

Luego de definida la estrategia se retomó el tema escogido: “Los grafitis como práctica cultural alternativa".

La planificación de la pregunta: ¿qué vamos a investigar? Ello condujo a la definición de los observables y su delimitación de espacio temporal. Esta identificación surge del enunciado del tema, por tanto, grafitis se erige como problema pero adjetivado como: práctica y como cultura alternativa. De manera que se trazan tres nudos problemáticos:

- grafitis

- Cultura

- práctica cultural alternativa

Este proceso de problematización del tema se realizó partiendo del sentido común. El objetivo era conocer y, con ello, poner en común, qué conocimientos se tenia sobre los grafitis. En ese ejercicio se tornaron evidente presupuestos; las ideas construidas a partir de la experiencia permitió establecer un horizonte común. De ese ejercicio surgió la pregunta: ¿quiénes son los que pintan? y ¿por qué lo hacen? Por tanto, de la problematización del tema a partir del sentido común, se pudo llegar a la primera enunciación del problema de investigación; es decir, el problema práctico. Esta acción condujo a planificación del trabajo de campo y la consecuente distribución de tareas. Para dar cuenta de ello, se identificaron los 3 niveles metodológicos y se plantearon las herramientas e instrumentos a utilizar. Cabe destacar que en esta perspectiva no se plantea la dicotomía entre lo cualitativo y lo 
cuantitativo. Por tanto, trasciende esa visión estanca y propone la mirada integral de cada uno de esos enfoques. Este posicionamiento reconoce que la perspectiva de investigación compleja revisten de un abordaje metodológico que de cuenta de ella. Por tanto, la construcción del dato como su comprensión son necesarios para andar este camino. En este sentido, vale citar a Pierre Bourdieu que en "La Distinción" expresa:

... no es posible justificar de manera unitaria y a la vez específica la infinita diversidad de las prácticas si no es a condición de romper con el pensamiento lineal, que conoce las estructuras simples de orden de la determinación directa, para dedicarse a la reconstrucción de las redes de las enmarañadas relaciones que se encuentran presentes en cada uno de los actores. (BOURDIEU, 2012, p. 122)

De manera que, el camino propuesto no unidireccional; por tanto, no parte del investigador - posicionado como ajeno al campo - y se dirige a los observables con una estrategia centrada en la constatación empírica o en la comprensión. Lo que aquí se trata, es de echar mano a todos los instrumentos, herramientas que el área problemática requiera para su análisis.

Teniendo en cuenta lo expresado, los niveles de análisis son:

\section{De primer orden o fenomenológico}

Este es el nivel de contacto directo con los observables. Este contacto se efectúa dentro de la realidad que los constituye y da forma. Es por ello, se considera que es el nivel de la vida cotidiana el que enviste de realidad a la practica investigadora. El contacto de primera mano. Es, por tanto, del orden del fenómeno. Las herramientas e instrumentos aquí comprendidos son:

- Entrevistas semiestructuradas

- Entrevistas en profundidad

- Focus group

- Observación participante

- Sondeo bibliográfico

El empleo de cada una de estas herramientas respondió a un orden de comprensión. En primer lugar se realizó la observación participante en un doble enfoque: 
a- individual (trabajo de campo)

b- grupal - zonal- (análisis de segundo orden)

\section{De segundo orden u objetivizante}

Una vez identificados y abordados los observables se procede al primer nivel de análisis. Según indica Pierre Bourdieu que este nivel refiere al de la construcción de las relaciones objetivas que estructuran tanto las prácticas como las representaciones de esas prácticas.

Dentro de este nivel se aplicaron las herramientas de:

- Mapeo / cartografiado

- Entrevista en profundidad

- Identificación de variables y codificación.

\section{De tercer orden o praxeológico}

Si bien es el último nivel ello se da en cuanto que propicia la meta- reflexión de lo realizado. Este no solo tiene como objeto el sistema de relaciones objetivas que construye el modo de conocimiento objetivista; este aborda las relaciones dialécticas entre esas relaciones objetivas y las disposiciones estructurales en las cuales ellas se actualizan. Este tipo de conocimiento establece un límite claro al punto de vista centrado en la objetividad y la objetivación que abordan a las prácticas de manera unilineal y externa. Ello se da, según Pierre Bourdieu, en lugar de construir el principio generador que se sitúa en el movimiento mismo de su realización.

El autor no desconoce la impronta de cada una de las maneras de conocimiento expresadas. Sin embargo, da un peso especial al praxeológico ya que: "... [este conocimiento] no anula las adquisiciones del conocimiento objetivista pero las conserva y amplía integrando lo que este conocimiento debió excluir para obtenerlas". (BOURDIEU, 2012, p. 184)

Este tipo de conocimiento conduce a no reducir la ciencia social a las estructuras objetivas pero, a su vez, impulsa a no perder de vista a dichas estructuras dado que la verdad de las experiencias residen en ella. Siendo así, el conocimiento praxeológico se distingue del fenomenológico dado que, si bien asume la evidencia del sentido común por una operación de construcción, establece una ruptura con todas las representaciones 
construidas; es decir, las clasificaciones dadas a priori.

Es aquí donde se inscribe la propuesta de investigación de Bourdieu. En ella sostiene que es en la construcción de las estructuras objetivas donde es posible asir los mecanismos donde se establecen las relaciones entre las estructuras y las prácticas o las representaciones que la acompañan.

\section{Conclusiones preliminares}

Este artículo, partió de las palabras de De Certeau cuando parafrasea a Freud sobre el hombre moderno y su malestar en la cultura. Decía que ya no reía, no había nada que lo condujera a sublimar su pesar en esta sociedad que lo ahora. Se partió desde ese punto porque se reconoce que es desde ese malestar en la cultura en el que nace la investigación en general y en comunicación, en particular.

En aras de asir eso que conduce y articula las prácticas de los agentes, las denominadas teorías de las comunicación esgrimieron un conjunto de propuestas teóricas y metodológicas. La mayoría de ellas fueron tomadas prestadas las teorías sociológicas, la psicología social, la antropología - entre otras -. Debido a ello, durante décadas se discutió si era posible afirmar la existencia de un objeto de la comunicación; por tanto, si era posible afirmar que los estudios de la comunicación respondieran a los cánones de la ciencia. Todo estaba en duda. Todo fue puesto en dudas. Es por ello que en este artículo se tomó la duda como un desafío epistemológico para así plantear un camino teórico y metodológico que permitiera colocar a la "comunicación social" en el lugar de la ciencia. Por tanto, se identificó un objeto de estudio, se recortó un corpus teórico y se plantearon caminos para dar cuenta del interrogante identificado.

Aquí indagar a los grafitis fue como abrir una puerta; puerta que condujo al estudio de la comunicación y la cultura. A la comunicación como práctica específicamente significante; como hacedora del orden social; como urdimbre en la cual se tejen los sentidos que articulan la vida cotidiana.

Queda pendiente para otro artículo contar cuáles son las trayectorias que trazan esos grafitis en el espacio social santacruceño. Quedaron muchas respuestas por dar. Queda mucho por tanto, hay que seguir; mientras haya preguntas habrá más por investigar. 


\section{BiBLIOGRAFÍA}

AMOZURRUTIA, José A. Complejidad y Ciencias Sociales. Un modelo adaptativo para la investigación interdisciplinaria. México: UNAM, 2011.

ARFUCH, Leonor. El espacio Biográfico. Dilemas de la subjetividad contemporánea. Buenos Aires: Fondo de Cultura Económica, 2002.

. La entrevista, una invención dialógica. Paidós estudios de comunicación. Buenos Aires, 2010.

BOOTH, C. Wayne. Cómo convertirse en un hábil investigador. México: GEDISA, 2004.

BOURDIEU, Pierre; PASSERON, J. C. Los herederos. Los estudiantes y la cultura. México: Siglo Veintiuno, 2003.

BOURDIEU, Pierre. Capital cultural, escuela y espacio social. 2ạ. Edición. México: Siglo Veintiuno, 2003.

. El sentido práctico. México: Siglo Veintiuno, 2007.

. El sentido social del gusto. Elementos para una sociología de la cultura. México: Siglo Veintiuno, 2010.

. (Comp.) La miseria del mundo. México: Fondo de Cultura económica.

BOURDIEU, Pierre; LOÏC WACQUANT. Una invitación a la sociología reflexiva. México: Siglo Veintiuno, 2005.

CASTORIADIS, Cornelius. La institución imaginaria de la sociedad. Paris: Editions du Seuil, 1975.

CASTORINA, José Antonio (Comp.) Representaciones sociales. Problemas teóricos y conocimientos infantiles. México: GEDISA, 1998.

DE CERTEAU, Michel. La invención de lo cotidiano. Artes de Hacer. México: Universidad Iberoamericana, 1996.

GALINDO CÁCERES, Luis Jesús. Ingeniería en Comunicación Social y Promoción Cultural. Sobre Cultura, cibercultura y redes sociales. Homo Sapiens. Universidad Nacional del Rosario, Benemérita Universidad Autónoma de Puebla, Rosario, 2011.

GONZÁLEZ, Jorge. Coordenadas del imaginario: protocolo para el uso de las cartografías culturales. Estudios sobre las culturas contemporáneas. México: Universidad de Colina, 1995.

. Digitalizados por decreto: cibercultur@ o inclusión forzada en América Latina. Estudios sobre las culturas contemporáneas. México: Universidad de Colina, 2008.

GUBERN, Román. Metamorfosis de la lectura. Barcelona: Anagrama, 2010.

JENKINS, Henry. Convergence Culture. La cultura de la convergencia de los medios de comunicación. México: Paidós comunicación, 2006.

JODELET, Denise. Les represéntatios socials. 7 edición. Paris: Presses Universitaries de France, 2003. 
. Folies et représentations sociales. Paris: Presses Universitaires de France, 2004.

LE GOFF, Jacques. El orden de la memoria. El tiempo como imaginario. Barcelona: Paidós, 1982.

MAAS, Margarita et all. Sociocibernética, cibercultur@ y sociedad. México: UNAM, 2012.

MARTÍN- BARBERO, JESÚS Y REY, Germán. Los ejercicios del ver. Hegemonía audiovisual y ficción televisiva. Barcelona: GEDISA, 1999.

MOSCOVICI Serge. El Psicoanálisis, su imagen y su público. Buenos Aires: Ed. Huemul, 1979.

MUSTERS, George. Vida entre los patagones. Un año de excursiones desde el estrecho de Magallanes hasta río Negro (1869-1870). Buenos Aires: Ediciones Continente, 2007.

NOVICK, Susana (comp.) Las migraciones en América Latina. Políticas, culturas y estrategias. Buenos Aires: CLACSO, 2008.

SARLO, Beatriz. La ciudad vista. Mercancías y cultura urbana. Buenos Aires: Siglo Veintiuno, 2009.

- Escenas de la vida posmoderna. Intelectuales, arte y videocultura en la Argentina. Buenos Aires: Seix Barral, 1994.

. La máquina Cultura. Buenos Aires: Seix Barral, 1998.

SENNET, Richard. La cultura del nuevo capitalismo. 3ạ. Edición. Anagrama ediciones, 2008.

SOTELO, Lucrecia. Hacia una construcción de las representaciones de la educación superior. UNPA, 2017, en prensa.

THOMPSON, John. Los media y la modernidad. Barcelona: Paidós, 1997.

WILLIAMS, Raymond. Marxismo y literatura. Buenos Aires: La cuarenta, 2009. 